\title{
Efecto del nitrógeno y el potasio sobre el intercambio gaseoso y la distribución de biomasa en albahaca (Ocimum basillicum $\mathrm{L}$.)
}

\section{Effect of nitrogen and potassium on gaseous exchange and biomass distribution in basil (Ocimum basillicum L.)}

ENRIQUE COMBATT C. ${ }^{1,2}$

DAIRO JAVIER PÉREZ P. ${ }^{1}$

ALFREDO JARMA-OROZCO'

Plantas del estudio con nutrientes $\mathrm{N}$ y K en albahaca.

Foto: J. Villalba A.

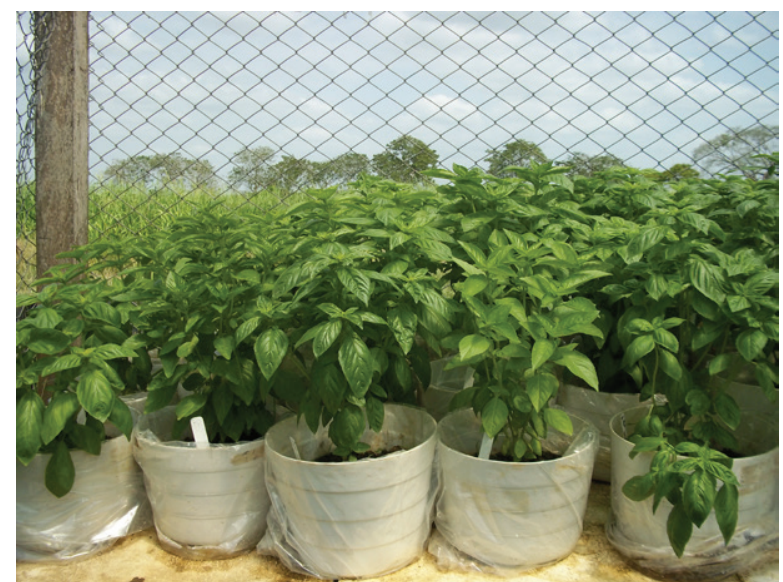

\section{RESUMEN}

La albahaca es considerada una planta promisoria de reciente explotación en Colombia, pero entre las limitaciones para su expansión, se encuentra la implementación de un plan de nutrición con la aplicación de nitrógeno y potasio, que permita el incremento y sostenibilidad de la producción. El objetivo del trabajo fue determinar el efecto de la fertilización con nitrógeno y potasio sobre variables de intercambio gaseoso, ganancia de biomasa e índice de cosecha (IC) de la albahaca (Ocimum basillicum L.) cv. Nufar F1. La investigación fue realizada en condiciones de umbráculo en la Facultad de Ciencias Agrícolas de la Universidad de Córdoba, Colombia. Se utilizó una matriz Box-Berard aumentada $3\left(2^{k}+2 k+2 k+1+1\right)$, para la obtención de combinaciones de nitrógeno y potasio. Las respuestas de intercambio gaseoso evaluadas fueron: tasa de fotosíntesis, conductancia estomática y concentración interna de $\mathrm{CO}_{2}$. En cuanto a biomasa, se evaluó masa seca de raíz, tallo, hoja e índice de cosecha. Los resultados más relevantes indicaron que las máximas tasas de fotosíntesis $\left(17,3 \mu \mathrm{mol} \mathrm{CO} \mathrm{m}^{-2} \mathrm{~s}^{-1}\right)$ fue observada aplicando $190 \mathrm{~kg} \mathrm{ha}^{-1} \mathrm{~N}$ y $12,5 \mathrm{~kg} \mathrm{ha}^{-1} \mathrm{~K}$, en tanto que la conductancia estomática fue mayor $\left(65,13 \mathrm{mmol} \mathrm{CO}_{2} \mathrm{~m}^{-2} \mathrm{~s}^{-1}\right)$, con la misma dosis de $\mathrm{N}$ pero con dosis de $\mathrm{K}$ de $237,5 \mathrm{~kg} \mathrm{ha}^{-1}$. Los valores más altos de masa seca de raíces, tallos y hojas fueron encontradas con las máximas dosis de $\mathrm{N}$ (190 $\left.\mathrm{kg} \mathrm{ha}^{-1}\right)$, aunque para el potasio las combinaciones que incrementaron la mayor acumulación de biomasa en raíces, tallos y hojas fueron 180,$69 ; 237,5$ y $12,5 \mathrm{~kg} \mathrm{ha}^{-1}$, respectivamente.

\footnotetext{
Palabras clave adicionales: nutrición mineral, plantas medicinales, variables fisiológicas, masa seca.

Facultad de Ciencias Agrícolas, Departamento de Ingeniería Agronómica y Desarrollo Rural, Universidad de Córdoba, Montería (Colombia). ORCID Combatt C., E.: 0000-0002-8760-0089; ORCID Pérez P., D.J.: 0000-0001-9041-5856; ORCID Jarma-Orozco, A.: 0000-0002-5821-2183

2 Autor para correspondencia.ecombatt@correo.unicordoba.edu.co.
} 


\section{ABSTRACT}

Basil is considered a promising plant of recent exploitation in Colombia, but among the limitations for its expansion, counts the implementation of a nutrition plan with the application of nitrogen and potassium, which result in increased and sustainable production. The objective was to evaluate the effect of nitrogen and potassium on the gaseous exchange variables and biomass gain and harvest index (HF) of a basil crop, hybrid Nufar $\mathrm{F}$. This research was carried out under greenhouse conditions in the Faculty of Agricultural Sciences of the University of Córdoba, Colombia. In order to optimize the response variables, a Box Bernard matrix was used, increased to 3 (2k $+2 \mathrm{k}+$ $2 \mathrm{k}+1+1)$ to obtain the combinations of nitrogen and potassium necessary for this research. Among the gaseous exchange variables evaluated were: the rate of photosynthesis, the stomatal conductance and the internal $\mathrm{CO}_{2}$ concentration. As for the biomass, the dry mass of the roots, stem, and leaves and the harvest index were evaluated. The most relevant results indicated that the maximum rate of photosynthesis $\left(17.3 \mu \mathrm{mol} \mathrm{CO} \mathrm{m}^{-2} \mathrm{~s}^{-1}\right)$ was observed when applying $190 \mathrm{~kg} \mathrm{ha}^{-1} \mathrm{~N}$ and $12.5 \mathrm{~kg} \mathrm{ha}^{-1} \mathrm{~K}$, while the stomatal conductance was higher $\left(65.13 \mathrm{mmol} \mathrm{CO}_{2} \mathrm{~m}^{-2}\right.$ $\mathrm{s}^{-1}$ ) with the same dose of $\mathrm{N}$ but with doses of $237.5 \mathrm{~kg} \mathrm{ha}^{-1}$ of $\mathrm{K}$. The highest value of the dry mass of the roots, stem and leaves was found with the maximum dose of $\mathrm{N}\left(190 \mathrm{~kg} \mathrm{ha}^{-1}\right)$ although, for potassium, the combinations that promoted the highest accumulation of biomass were differential for the roots, stem and leaves $(180.69,237.5$ and $12.5 \mathrm{~kg} \mathrm{ha}^{-1}$, respectively).

Additional key words: mineral nutrition, medicinal plants, physiological variables, dry mass.

Fecha de recepción: 16-05-2017 Aprobado para publicación: 30-01-2018

\section{INTRODUCCIÓN}

La albahaca (Ocimum basillicum L.) es una planta aromática y medicinal compuesta por altos contenidos de aceites esenciales tales como monoterpenos, sesquiterpenos y sus análogos oxigenados, presentes en bajas concentraciones (Búfalo et al., 2015), utilizada en elaboración de alimentos, fármacos y perfumes; además es usada en medicina tradicional y en otras costumbres populares de las regiones productoras ( $\mathrm{Pa}-$ checo y Jürgen, 2005). Según Koba et al. (2009), las hojas frescas y secas de albahaca se utilizan como aromáticas, aceites esenciales, y sus componentes principales también se utilizan como fármacos vegetales, ya que tiene actividad antimicrobiana y fungistática.

La albahaca y otras especies aromáticas son cultivos promisorios de reciente explotación en Colombia, consideradas recursos genéticos de gran potencial económico, los cuales cada vez más atraen nuevos inversionistas por su reciente entrada y comercialización a mercados internacionales. En la actualidad las principales áreas se encuentran en los departamentos de Putumayo y Boyacá (20 ha cada uno), Cundinamarca (9 ha) y Tolima (8 ha), con rendimientos promedio de 20, 27, 24 y $19 \mathrm{t} \mathrm{ha}^{-1}$ (Agronet, 2017). Según Zheljazkov et al. (2008), la albahaca dulce $(O$. basillicum) es una de las especies considerada como promisorias para el aprovechamiento industrial de su aceite esencial, dado que posee una producción mundial estimada en 43 t de aceite albahaca al año con un valor de 2,8 millones de US\$.

Debido al reciente incremento en la explotación y expansión del cultivo en Colombia, se ha evidenciado que existen serias limitaciones para que el rendimiento esté acorde con los requerimientos de calidad exigidos por los mercados internacionales. Lo anterior puede ser una consecuencia de las diversas y deficientes tecnologías de manejo, entre ellas la aplicación de dosis de nutrientes o elementos químicos esenciales, que contribuyan a la producción de volúmenes constantes y de altos estándares de calidad (Bonilla y Guerrero, 2010).

En términos generales, la utilización eficiente de nitrógeno y potasio, es una meta esencial en el manejo de los cultivos (Patil, 2010); los estudios de nutrición deben estar dirigidos al establecimiento de la relación entre el suministro de nutrientes y el crecimiento de las plantas en términos de producción de materia seca (Marschner, 2002) y eficiencia fotosintética. 
Un aspecto importante en la nutrición mineral, cuya deficiencia afecta la cantidad y calidad del rendimiento en albahaca, y en muchos cultivos, son los niveles de fertilización nitrogenada y potásica, entre otras. Matsumoto et al. (2013), verificaron que existe interacción entre $\mathrm{N}$ y K en el incremento del área foliar; los autores indican que, aplicaciones elevadas de $\mathrm{N}$ y bajas de $\mathrm{K}$, incrementaron de manera importante el área foliar, en tanto que al aumentar la dosis de estos dos elementos, no se observó un incremento en esta variable. Biesiada y Kuś (2010), en un estudio sobre nutrición en albahaca encontraron altas cantidades de P y Mg foliar, con la aplicación edáfica de $150 \mathrm{~kg}$ ha ${ }^{-1}$ de $\mathrm{N}$, mientras que altas cantidades de $\mathrm{K}$ y Ca fueron verificados con $250 \mathrm{~kg} \mathrm{ha}^{-1}$ de este mismo elemento. Por su parte, Sifola y Barbieri (2006), indican que la fertilización nitrogenada correlaciona directamente con el crecimiento, rendimiento y contenido de aceite esencial de albahaca.

Pereyra (2001), indica que la constitución estructural de los aminoácidos no sólo está dada por el requerimiento de esqueletos carbonados sintetizados en la fotosíntesis para la incorporación del amonio, sino también debido a la dependencia de algunas reacciones del metabolismo del nitrógeno de ATP y NADPH, producido en la fotosíntesis. Por otra parte, en los tejidos no fotosintéticos, los requerimientos energéticos son obtenidos por la degradación de los azúcares transportados desde las hojas.

Según Taiz y Zeiger (2006), el potasio $\left(\mathrm{K}^{+}\right)$funciona como activador de enzimas esenciales para la fotosíntesis y la respiración, además es uno de los contribuyentes más importantes del potencial osmótico de las células y por consiguiente, a su presión de turgencia, siendo fundamental en el balance de las cargas negativas de ácidos orgánicos y de aniones tales como el sulfato y nitrato, absorbidos por las raíces del medio externo y manteniendo la electro-neutralidad de las células.

Actualmente no existen reportes de dosis óptimas de fertilización con $\mathrm{N} \mathrm{y} \mathrm{K}^{+}$en cultivos de albahaca bajo la oferta ambiental del Sinú en Córdoba, Colombia. Esta situación limita la expresión del máximo potencial en rendimiento y aumenta su susceptibilidad a plagas y enfermedades, lo que finalmente incide en la producción de hojas de baja calidad, que no cumplen con los estándares de comercialización exigidos por mercados internacionales. Además los ingresos y competitividad de este cultivo son bajos, lo que compromete su sostenibilidad y la posibilidad de mejora de calidad de vida de los medianos y pequeños productores.

El objetivo de la presente investigación fue determinar el efecto del $\mathrm{N}$ y $\mathrm{K}$ sobre algunos aspectos de intercambio gaseoso y distribución de biomasa en albahaca establecida bajo condiciones controladas, con el propósito de construir conocimiento para incrementar la productividad de la especie y ofrecer alternativas de producción al sector agrícola del departamento de Córdoba.

\section{MATERIALES Y MÉTODOS}

La investigación fue realizada bajo condiciones controladas en casa de vegetación del Centro Experimental de la Facultad de Ciencias Agrícolas de la Universidad de Córdoba, ubicada en Montería, Colombia (8॰48' N y 78॰53' W), clasificación climática de Bosque seco tropical (BsT), con promedio anual de temperatura de $27,4^{\circ} \mathrm{C}$, precipitación de $1.346 \mathrm{~mm}$ año- ${ }^{-1}, 85 \%$ de humedad relativa y $2.108 \mathrm{~h}$ luz por año (Palencia et al., 2006).

Se utilizó una matriz Box-Berard aumentada 3 (Box, 1954), definida por la siguiente expresión: $2 k+2 k+2 k+1+1$, en donde $k$ corresponde al número de factores involucrados (Tab. 1); se obtuvieron catorce (14) tratamientos, mediante la combinación de siete dosis de nitrógeno $(10 ; 25 ; 50 ; 100 ; 150 ; 175$ y $\left.190 \mathrm{~kg} \mathrm{ha}^{-1}\right)$ y siete de potasio $(12,5 ; 31,25 ; 62,5 ; 125$; 187,$5 ; 218,8$ y $237,5 \mathrm{~kg} \mathrm{ha}^{-1}$ ). Se usó un diseño completamente al azar con un arreglo factorial parcial y tres repeticiones.

Cada unidad experimental fue constituida por una planta del híbrido Nufar F1 establecida en una maceta de $6 \mathrm{~kg}$ de capacidad, que contenía un suelo de textura arcillo limosa, típico del Valle del Sinú medio, con las siguientes características químicas: $6,6 \mathrm{de} \mathrm{pH}$; CICe 21,9 $\mathrm{cmol}^{(+)} \mathrm{kg}^{-1}$; materia orgánica 3,96\%; azufre y fósforo disponible con 10,4 y 12,6 $\mathrm{mg} \mathrm{kg}^{-1}$, además 13,2; 7,9; 0,55 y 0,20 $\mathrm{cmol}^{(+)} \mathrm{kg}^{-1}$ de $\mathrm{Ca}, \mathrm{Mg}, \mathrm{K}$ y $\mathrm{Na}$, respectivamente. Los micronutrientes $\mathrm{Cu}, \mathrm{Fe}, \mathrm{Zn}$, Mn y B se encontraron en cantidades de 2,8; 27,2; 4,0; 60,8 ; y $0,28 \mathrm{mg} \mathrm{kg}^{-1}$, respectivamente.

La fertilización fue realizada a través de la aplicación de soluciones nutritivas de $\mathrm{N}$, K y elementos menores (B, Zn, Cu, Mn y Mo). El $\mathrm{N}$ fue suministrado en forma de $\mathrm{NH}_{4}$ y fraccionado en tres partes iguales a los 10, 44 y 77 días después del trasplante. El K fue 


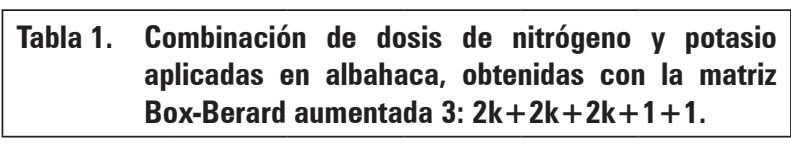

\begin{tabular}{|c|c|c|c|c|c|}
\hline \multirow[t]{2}{*}{ Tratamiento } & \multicolumn{2}{|c|}{$\begin{array}{l}\text { Variables } \\
\text { codificadas }\end{array}$} & \multicolumn{2}{|c|}{$\begin{array}{l}\text { Nutriente } \\
\left(\mathrm{kg} \mathrm{ha}^{-1}\right)\end{array}$} & \multirow[t]{2}{*}{ Expresiór } \\
\hline & $X_{1}$ & $X_{2}$ & $\mathrm{~N}$ & $\mathrm{~K}$ & \\
\hline 1 & -1 & -1 & 50 & 62,5 & \multirow{4}{*}{$2 k$} \\
\hline 2 & -1 & 1 & 50 & 187,5 & \\
\hline 3 & 1 & -1 & 150 & 62,5 & \\
\hline 4 & 1 & 1 & 150 & 187,5 & \\
\hline 5 & $-1,5$ & 0 & 25 & 125 & \multirow{4}{*}{$2 k$} \\
\hline 6 & 1,5 & 0 & 175 & 125 & \\
\hline 7 & 0 & $-1,5$ & 100 & 31,25 & \\
\hline 8 & 0 & 1,5 & 100 & 218,8 & \\
\hline 9 & $-1,8$ & -1 & 10 & 62,5 & \multirow{4}{*}{$2 k$} \\
\hline 10 & -1 & $-1,8$ & 50 & 12,5 & \\
\hline 11 & 1,8 & 1 & 190 & 187,5 & \\
\hline 12 & 1 & 1,8 & 150 & 237,5 & \\
\hline 13 & 0 & 0 & 100 & 125 & $1^{*}$ \\
\hline 14 & $-1,8$ & $-1,8$ & 10 & 12,5 & $1^{*}$ \\
\hline
\end{tabular}

*Tratamiento adicional.

aplicado en forma de $\mathrm{KCl}$ y fraccionado en dos aplicaciones iguales, las cuales coincidieron con la primera y segunda fertilización de $\mathrm{N}$. Con el fin de evitar deficiencias nutricionales que pudieran influir sobre la respuesta de $\mathrm{N}$ y $\mathrm{K}$, se realizó una aplicación uniforme de $\mathrm{P}$ fraccionada en dos partes iguales, la primera un día antes del trasplante y la segunda con el 50\% de la solución de micronutrientes; la segunda aplicación de micronutrientes se realizó con la segunda fertilización de $\mathrm{N}$ y K.

En el período de cosecha (inicio de floración), se evaluaron variables de intercambio gaseoso: fotosíntesis neta $(P n)$, concentración interna de $\mathrm{CO}_{2}$ en la cámara subestomática $\left(C_{i}\right)$ y la conductancia estomática $\left(g_{s}\right)$. Las mediciones fueron realizadas con un Analizador de Gases al Infrarrojo (IRGA) modelo CIRAS 2 (PP Systems, Hitchin, UK), tomando la quinta hoja en sentido descendente (sana y fotosintéticamente activa); las lecturas fueron realizadas entre las 9:00 y 11:00 am utilizando estándares de la radiación fotosintéticamente activa (RFA) en $1.000 \mu$ moles de fotones $\mathrm{m}^{-2} \mathrm{~s}^{-1} \mathrm{y}$ concentración de $\mathrm{CO}_{2}$ en 350 ppm.

La cuantificación de biomasa de raíces, tallos y hojas, se realizó al momento de la cosecha sometiendo las muestras a $72^{\circ} \mathrm{C}$ durante $3 \mathrm{~d}$; el área foliar se determinó con el software libre ImageJ (versión 1.45).

Los efectos de los tratamientos fueron evaluados con ecuaciones de regresión lineal múltiple en función de $\mathrm{N}$ y K, mediante las cuales se determinaron las dosis óptimas de estos elementos y la máxima respuesta de las variables. Las ecuaciones de regresión se determinaron con el programa R v. 3.2.2 (R Development Core Team, 2015), las cuales fueron seleccionadas en función de la significancia de los coeficientes de los parámetros de las ecuaciones y del coeficiente de determinación $\left(R^{2}\right)$, además de su funcionalidad entre las dosis de nutrientes aplicados; asimismo, fueron representados en superficies de respuesta para mejorar su análisis e interpretación.

\section{RESULTADOS Y DISCUSIÓN}

\section{Fotosíntesis y conductancia estomática.}

Los máximos valores de la $P n$ y $g_{s}$, fueron explicadas por modelos de regresión de segundo orden (Tab. 2), observando un importante efecto de las altas dosis de $\mathrm{N}$ en ambas respuestas, en tanto que altas dosis de $\mathrm{K}$ fueron más importantes para la conductancia. Lo anterior confirma la fuerte relación entre la asimilación del carbono y el N. Según Azcón-Bieto y Talón (2008), la albahaca, que pertenece al grupo de plantas de fotosíntesis tipo C3, requiere de una alta cantidad de $\mathrm{N}$ para aumentar la concentración de ribulosa 1,5 bifosfato carboxilasa-oxigenasa (rubisco) en las hojas, principalmente como consecuencia de un incremento de la proteína soluble y el nitrógeno total y así mantener altas tasas de $P n$.

Por otra parte, Pessarakli (2001), reporta que existe una correlación positiva entre la $P n$ y el contenido de $\mathrm{N}$ en la hoja y que la rubisco y otras enzimas de asimilación del $\mathrm{CO}_{2}$ y del metabolismo de carbohidratos, hacen más efectiva la captación de energía lumínica. En ese sentido, Pereyra (2001) resalta la evidente relación entre el $\mathrm{N}$ y la regulación de la actividad y síntesis de las enzimas del metabolismo del carbono. La autora indica que la luz no solo afecta la fotosíntesis sino que también controla la expresión de la nitrato reductasa (NR), nitrito reductasa (NiR) y la sacarosa fosfato sintetasa (SPS), de tal manera que la acumulación de los productos finales de la fotosíntesis aumentan la actividad de estas enzimas, existiendo un rápido ajuste del ritmo de reducción del nitrato 
a fluctuaciones en la disponibilidad de carbohidratos, regulando en definitiva, los flujos del carbono $\left(P n y g_{s}\right)$ y nitrógeno en las células vegetales.

En cuanto al $\mathrm{K}$, aunque en este trabajo se observó un mayor efecto de este elemento sobre la $g_{s}$ respecto a la $P n$, es importante mencionar que este mineral es esencial para la activación y síntesis de ATP y proteínas asociadas con la fijación del $\mathrm{CO}_{2}$ y que, cantidades considerables en los estomas permiten mantener altas tasas de $P n$, debido a la regulación de su apertura y la reducción de los niveles de ácido abscísico en la planta, permitiendo mayores tasas de $g_{s}$ (Marschner, 2002; Mejía, 2010). Consecuentemente con lo anterior Barker y Pilbeam (2007), reportaron que incrementos en la asimilación de $\mathrm{CO}_{2}$, acompañados de un decrecimiento en la respiración mitocondrial, fueron correlacionados con incrementos en la concentración de $\mathrm{K}$ en la hoja. Los autores indican además que el estado energético de la planta está estrechamente relacionado con el transporte de iones $\mathrm{K}$ a través de las membranas del cloroplasto y la mitocondria y que este elemento tiene una influencia favorable sobre la fotorreducción y la fotofosforilación.

Con base en la respuesta de la $g_{s}$, se encuentra en función de altas dosis de $\mathrm{N}$ y $\mathrm{K}$ (Tab. 2). De acuerdo con el modelo de regresión $\hat{\mathrm{Y}}=89,5217$ $-0,7813 \mathrm{~N}^{* *}+0,1634 \mathrm{~K}+0,0049 \mathrm{~N}^{2 * *}-0,0016 \mathrm{NK}^{*}$, se determinó que, para llegar al máximo valor de la $g_{s}$ en albahaca $\left(65,13 \mathrm{mmol} \mathrm{CO} \mathrm{m}^{-2} \mathrm{~s}^{-1}\right)$, se necesitan $190 \mathrm{y}$ $237,5 \mathrm{~kg} \mathrm{ha}^{-1}$ de $\mathrm{N}$ y $\mathrm{K}$, respectivamente. En ese sentido, Jákli et al. (2017) indican que con frecuencia la $g_{s}$ se reduce bajo deficiencia de K; sin embargo, los autores indican que algunos estudios cuestionan la causalidad directa entre la deficiencia de K y el cierre estomático. Por lo tanto, una disminución de la $g_{s}$ no reflejaría necesariamente una baja capacidad para el control de apertura y cierre estomático, pero podría ser el resultado de un ajuste de los estomas a la reducción de la utilización de $\mathrm{CO}_{2}$ por la fotosíntesis.
En relación a la concentración interna de $\mathrm{CO}_{2}(\mathrm{C} i)$ en la cámara subestomática, se encontró que esta concentración depende en mayor proporción de los niveles de potasio respecto a los de nitrógeno (Fig. 1); la $C i$ se incrementó significativamente con las dosis de $\mathrm{K}$ hasta su punto de saturación, lo cual fue explicado por el modelo $\hat{\mathrm{Y}}=45,4173-0,3693 \mathrm{~N}+1,2429 \mathrm{~K}$ $0,0049 \mathrm{~K}^{2}\left(R^{2}=49,41 \%\right)$. Mediante esta ecuación se determinó que la dosis máxima de potasio para encontrar la máxima $C i$ en albahaca fue de $127,02 \mathrm{~kg}$ $\mathrm{ha}^{-1}$, en tanto que para el N, las más altas Ci $(120,54$ ppm de $\mathrm{CO}_{2}$ ), se observaron con dosis de $10 \mathrm{~kg} \mathrm{ha}^{-1}$. En esta figura también se observa que existe una relación inversamente proporcional entre la dosis de $\mathrm{N}$ y la $C i$, lo que podría explicarse por la mayor actividad de rubisco con las altas dosis de $\mathrm{N}$ que agotarían rápidamente la $C i$; asimismo se encontró que dosis intermedias de potasio incrementan la concentración interna de $\mathrm{CO}_{2}$, resultados que confirman que la nutrición con $\mathrm{K}$ contribuye con la asimilación del $\mathrm{CO}_{2}$, tal como lo reportan Azcón-Bieto y Talón (2008).

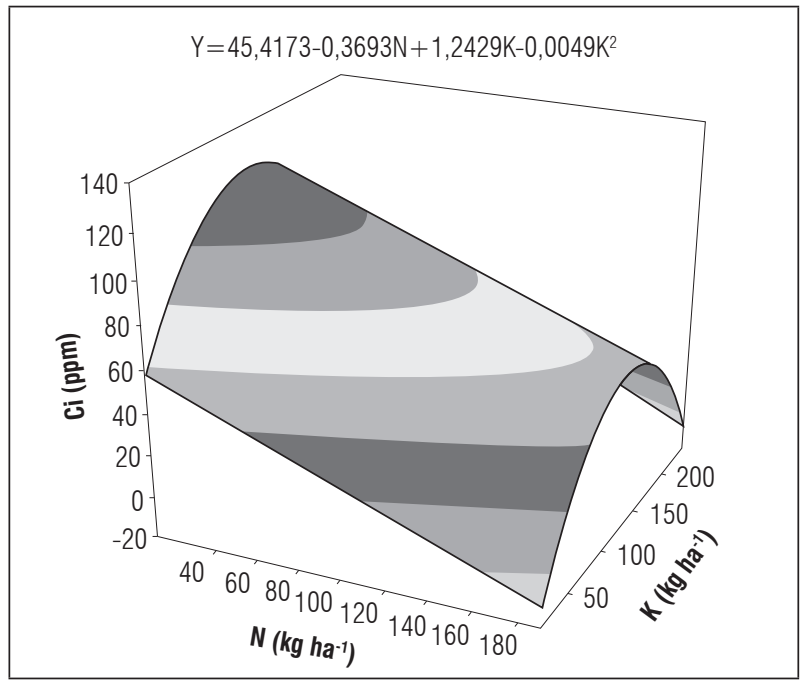

Figura 1. Concentración interna de $\mathrm{CO}_{2}$ (Ci) en función de las dosis de $\mathbf{N}$ y $\mathrm{K}$ en el cultivo de albahaca.

Tabla 2. Modelos de regresión y máxima respuesta de la fotosíntesis $(P n)$ y la conductancia estomática $\left(g_{s}\right)$ de albahaca $(0$. basillicum) en función de las dosis óptimas de $\mathrm{N}$ y $\mathrm{K}$.

\begin{tabular}{|c|c|c|c|c|c|c|}
\hline \multirow{2}{*}{ Variable } & Modelo de regresión & \multirow{2}{*}{$R^{2}$} & \multirow{2}{*}{ CV (\%) } & \multicolumn{2}{|c|}{ Dosis $\left(\mathrm{kg} \mathrm{ha}{ }^{-1}\right)$} & \multirow{2}{*}{ Máxima respuesta } \\
\cline { 5 - 8 } & & & & $\mathrm{N}$ & $\mathrm{K}$ & \\
\hline$P_{n}$ & $\hat{\mathrm{Y}}=16,2761-0,1254 \mathrm{~N}^{* *}-0,0334 \mathrm{~K}+0,0007 \mathrm{~N}^{2 *}+0,0001 \mathrm{~K}^{2}$ & 0,40 & 22,49 & 190 & 12,50 & $17,31 \mu \mathrm{mol} \mathrm{CO}_{2} \mathrm{~m}^{-2} \mathrm{~s}^{-1}$ \\
\hline$g_{s}$ & $\hat{\mathrm{Y}}=89,1-0,7813 \mathrm{~N}^{* *}+0,1634 \mathrm{~K}+0.0049 \mathrm{~N}^{2 *}-0.0016 \mathrm{NK}^{*}$ & 0,41 & 20,21 & 190 & 237,5 & $65,13 \mathrm{mmol} \mathrm{CO}_{2} \mathrm{~m}^{-2} \mathrm{~s}^{-1}$ \\
\hline
\end{tabular}

${ }^{*}$ : significativo al $5 \% ;{ }^{* *}$ : altamente significativo al $1 \%$. 


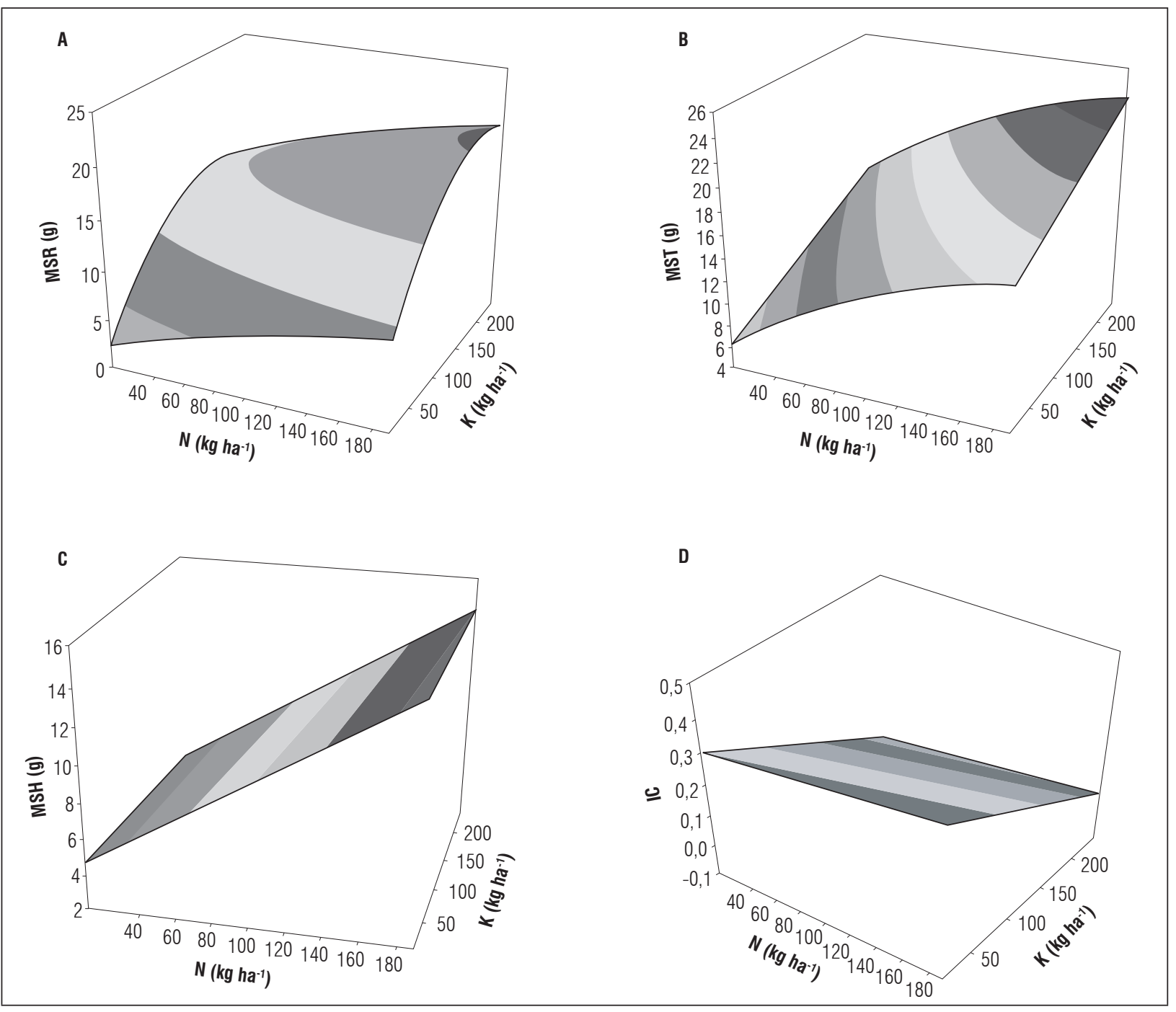

Figura 2. Superficie de respuesta de la biomasa de masa seca de raíz-MSR (A), tallo-MST (B), hojas-MSH (C) e índice de cosecha-IC (D), en función de la aplicación de dosis de $\mathrm{N}$ y $\mathrm{K}$ en el cultivo de albahaca.

Respecto a la masa seca de raíces, tallos, hojas e índice de cosecha, se pudo determinar que estas respuestas se ajustaron a ecuaciones de segundo orden, generalmente explicadas por el incremento significativo en ganancia de masa seca en la medida que se presentaron aplicaciones crecientes de $\mathrm{N}$ y K (Fig. 2). El K junto al $\mathrm{N}$ y $\mathrm{P}$, son conocidos como los "tres factores esenciales" de la nutrición mineral de las plantas (Hu et al., 2016). Robertson et al. (2009), explican que para plantas medicinales, es necesario conocer los niveles óptimos de fertilización que permitan proveer suficiente $\mathrm{N}$ a la planta para maximizar su crecimiento, rendimiento y cantidad de compuestos benéficos para la salud humana.
Los resultados del presente trabajo no sorprenden si se considera la estrecha relación sinérgica de estos elementos; una deficiencia de $\mathrm{K}$ podría afectar significativamente el metabolismo del $\mathrm{N}$, especialmente el metabolismo de aminoácidos y proteínas. Wang et al. (2012), indican que el metabolismo del $\mathrm{N}$ es determinante en el metabolismo de la planta y que se ha observado que las deficiencias de $\mathrm{K}$ causan una sustancial reducción en la asimilación del $\mathrm{N}$ y reducen la asimilación radicular del $\mathrm{NO}_{3}^{-}$. Se ha demostrado que el suministro de $\mathrm{K}$ aumenta la absorción del $\mathrm{NO}_{3}{ }_{3}$ en raíces, así como el transporte desde la raíz hasta el dosel, por lo que es necesario centrarse en el efecto que la deficiencia de K ejerce sobre el metabolismo de 
$\mathrm{N}$ en la hoja y en general en la planta, y no sólo en la raíz (Hu et al., 2016).

\section{Masa seca de raíz (MSR)}

La respuesta de la MSR, fue explicada por una tendencia positiva creciente a la dosis de $\mathrm{N}$, independientemente de la dosis de K; se evidenció la máxima respuesta con niveles de $\mathrm{N}$ y K de 190 y 180,69 kg $\mathrm{ha}^{-1}$, respectivamente, para obtener un rendimiento de 20,67 g de biomasa radicular por planta (Fig. 2A). El modelo que explicó esta tendencia fue $\hat{\mathrm{Y}}=$ - $0,1699+0,0583 \mathrm{~N}+0,1444 \mathrm{~K}^{* *}-0,0001 \mathrm{~N}^{2}-0,0004 \mathrm{~K}^{2 * *}$ $\left(R^{2}=75,06 \%\right)$. El efecto de la aplicación de $\mathrm{N}$ sobre el crecimiento de las raíces es poco entendido, pero, tal como se discutió anteriormente, es evidente el mayor crecimiento de estas cuando el nitrógeno no es limitante, lo que además podría explicarse por la proliferación de raicillas (datos no mostrados), o el aumento en la longitud radical en la zona de aplicación del fertilizante, lo que potenciaría la absorción de otros elementos en horizontes más profundos del suelo.

\section{Masa seca del tallo}

La masa seca del tallo (MST) se incrementó positivamente conforme aumentaron las dosis de $\mathrm{N}$ y K; el modelo de regresión $\hat{\mathrm{Y}}=4,8714+0,0974 \mathrm{~N}+0,0308 \mathrm{~K}$ $0,0002 \mathrm{~N}^{2}\left(R^{2}=79,00\right)$, indica que la máxima ganancia de MST se presentó con las aplicaciones de $190 \mathrm{~kg} \mathrm{ha}^{-1}$ de $\mathrm{N}$ y $237,5 \mathrm{~kg} \mathrm{ha}^{-1}$ de K (Fig. 2B). Estos resultados coinciden con los reportes de Cenóz y Burgos (2005), quienes al evaluar dosis entre 150 y $300 \mathrm{~kg} \mathrm{ha}^{-1}$ de N en forma de triple 15 en albahaca, obtuvieron las mayores ganancias de biomasa de tallo cuando el suministro del fertilizante fue mayor. Como se mencionó, la ganancia de biomasa en el tallo de las plantas sometidas a las mayores dosis de $\mathrm{N}$ y K, podría ser una consecuencia del efecto sinérgico de estos elementos. Sharafzadeh y Alizadeh (2011) verificaron una mayor acumulación de masa seca de brotes de albahaca cuando fueron aplicadas dosis altas de NPK con 150, 100 y $100 \mathrm{~kg} \mathrm{ha}^{-1}$, respectivamente. Barker y Pilbeam (2007), indican que el $\mathrm{K}$ es requerido en grandes cantidades por la planta por su papel fundamental en la retraslocación de fotoasimilados y en el incremento de la turgencia de las células, lo que genera presión para el crecimiento de tallos y hojas, influenciando finalmente el rendimiento y la calidad final. Por su parte, Sifola y Barbieri (2006) al evaluar el efecto de la fertilización nitrogenada sobre la cantidad y calidad de aceites esenciales en albahaca, encontraron una relación positiva entre el $\mathrm{N}$ y estas dos respuestas, lo que atribuyeron al aumento en la cantidad de biomasa por unidad de superficie, al desarrollo del área foliar y el aumento de la tasa fotosintética.

\section{Masa seca de hojas (MSH)}

La máxima ganancia de MSH está asociada principalmente con las aplicaciones de $\mathrm{N}$ y el modelo que explicó esta tendencia fue $\hat{\mathrm{Y}}=4,1226+0,0568 \mathrm{~N}^{* *}$ $0,0032 \mathrm{~K}+0,00001 \mathrm{~K}^{2}\left(R^{2}=84,87 \%\right)$, mediante el cual se encontró que la dosis máxima aplicada de $\mathrm{N}$ con $190 \mathrm{~kg} \mathrm{ha}{ }^{-1}$ y mínina de $\mathrm{K}$ con $12,5 \mathrm{~kg} \mathrm{ha}^{-1}$, son las que influyen en los máximos rendimientos de esta variable con 14,87 g (Fig. 2C). Estos resultados son contrarios a los reportados por Jaćimović et al. (2010), quienes no encontraron efecto del aumento de las dosis de $\mathrm{N}$ en la ganancia de masa seca de hoja en albahaca dulce, pero similares a los encontrados por Nurzyńska-Wierdak et al. (2012) quienes no encontraron ningún efecto significativo de la dosis de potasio sobre las características biométricas examinadas en las plantas de albahaca.

La alta demanda de $\mathrm{N}$ se explica por la función que cumple este elemento en las hojas, ya que de acuerdo a Pessarakli (2001), es indispensable para el desarrollo del aparato fotosintético, a través de enzimas, pigmentos y otros componentes requeridos para incrementar la capacidad asimilatoria de la planta, la cual es retribuida por una mayor intercepción de luz y fotosíntesis de dosel, lo que conlleva a mayores tasas de crecimiento y rendimiento.

Las respuestas variables del $\mathrm{K}$ podrían explicarse por su efecto sobre el metabolismo de proteínas y aminoácidos que al parecer, dependen del tipo de planta (Hu et al., 2016); algunos autores indican que este metabolismo está conectado con la actividad cambiante de la nitrato reductasa (NR) (Wang et al., 2012) y la glutamina sintetasa (GS) (Ding et al., 2006) ante deficiencias de $\mathrm{K}$.

Los resultados encontrados en esta investigación, se aproximan a los reportes de Matsumoto et al. (2013) en $O$. basillicum, quien encontró que la masa seca aérea en función del $\mathrm{N}$ mostró una tendencia cuadrática, donde dosis de $\mathrm{N}$ cercanas a los $70 \mathrm{~kg} \mathrm{ha}^{-1}$ produjeron hasta $35 \mathrm{~g}$ por planta. Según reportes de Cenóz y Burgos (2005), en O. basillicum, las mayores ganancias de biomasa se obtienen con dosis de 150 a $300 \mathrm{~kg} \mathrm{ha}^{-1}$ de $\mathrm{N}$ en forma de triple 15; Golcz et al. (2006) en un estudio sobre fertilización en el cultivo de albahaca dulce, indicaron que dosis intermedias de 
$\mathrm{N} /$ planta $(1,2 \pm 0,6 \mathrm{~g})$, resultan en una mayor nivel de clorofila foliar y mayores tasas de fotosíntesis; por su parte Nguyen et al. (2010), demostraron que los cambios en el nivel de potasio afectan significativamente la composición de los fenoles y la capacidad antioxidante de las hojas de albahaca.

\section{Índice de cosecha (IC)}

Consecuentemente con lo observado en las hojas, los altos IC se observaron con aplicaciones elevadas de $\mathrm{N}$ y bajas de $\mathrm{K}$, los cuales fueron determinados por el modelo estadístico $\hat{\mathrm{Y}}=0,3171+0,0005 \mathrm{~N}^{* *}$ $0,0015 \mathrm{~K}^{* *}+0,0000 \mathrm{~K}^{2 * *} \quad\left(R^{2}=54,17 \%\right)$, con dosis de $\mathrm{N}$ y K de 190 y 12,5 kg ha-1, respectivamente (Fig. 2D). Estos resultados difieren del descrito por Rincón (2002) en el cultivo de lechuga icelberg, quien indica que el IC se reduce en la medida que se incrementan las dosis de $\mathrm{N}$ especialmente por encima de dosis superiores a $100 \mathrm{~kg} \mathrm{ha}^{-1}$. Según Malik et al. (2011), la plantación de hierbas medicinales bajo manejo integrado de fertilizantes orgánicos o inorgánicos, es la mejor estrategia actual que pueden emplearse para mejorar el rendimiento y los compuestos activos de estas plantas. Wahab y Hornok (1982) encontraron que un incremento de las dosis de $\mathrm{N}$ proporcionó un aumento en el rendimiento fresco y seco de la albahaca dulce y Frabboni et al. (2011) al estudiar el efecto de dosis de nitrógeno sobre variables fisiológicas como área foliar, número de ramificaciones por planta y biomasa fresca de hojas, encontraron la mejor respuesta en rendimientos a dosis de $160 \mathrm{~kg} \mathrm{ha}^{-1} \mathrm{de} \mathrm{N}$. Ferreira et al. (2016) al evaluar las características productivas del cultivo de albahaca verificaron que las dosis de nitrógeno entre 90 a $135 \mathrm{~kg} \mathrm{ha}^{-1}$ fueron las más adecuadas, porque elevaron todas las características productivas del cultivo.

Aumentos en el IC en albahaca, ante elevadas dosis de $\mathrm{N}$, podrían estar explicados además por el aumento en el número de hojas por planta, ya que las plantas que crecen bajo condiciones deficientes de este elemento, reducirían el número de hojas para mantener la concentración de $\mathrm{N}$ adecuada en las hojas que quedan en el dosel, tal como se ha demostrado también en papa (Sifola y Barbieri, 2006).

\section{CONCLUSIONES}

Para la producción comercial de albahaca en el departamento de Córdoba, la aplicación de nitrógeno es fundamental para el incremento de la tasa de fotosíntesis y el potasio para la conductancia estomática, lo que se traduce en una mayor eficiencia fisiológica y/o producción de biomasa.

La albahaca demanda altas dosis de $\mathrm{N}$ y K para la producción de raíces y tallos, dado que la máxima respuesta en masa seca de raíz se registró con los niveles óptimos de $190 \mathrm{~kg}$ de $\mathrm{N}$ y 180,69 kg ha-1 de K; y para la masa seca de tallo con dosis de $190 \mathrm{~kg} \mathrm{ha}^{-1}$ de $\mathrm{N} \mathrm{y}$ $237,5 \mathrm{~kg} \mathrm{ha}^{-1}$ de K. En cambio, la producción de masa seca de hojas e índice de cosecha están asociadas principalmente con las aplicaciones de nitrógeno (dosis óptima de $190 \mathrm{~kg} \mathrm{ha}^{-1}$ ).

Conflicto de intereses: el manuscrito fue preparado y revisado con la participación de los autores, quienes declaran no tener algún conflicto de interés que coloquen en riesgo la validez de los resultados aquí presentados.

\section{REFERENCIAS BIBLIOGRÁFICAS}

Agronet. 2013. Base de datos de estadísticas agrícolas: área, producción, rendimiento y participación. En: http:// www.agronet.gov.co; consulta: enero de 2016.

Azcón-Bieto, J. y M. Talón. 2008. Fundamentos de fisiología vegetal. $2^{a}$ ed. Editorial Mc Graw Hill Interamericana, Madrid, España.

Barker, A. y D. Pilbeam (eds.). 2007. Handbook of plant nutrition. CRC Taylor \& Francis Group, Boca Raton, FL, USA..

Biesiada, A. y A. Kuś. 2010. The effect of nitrogen fertilization and irrigation on yielding and nutritional status of sweet basil (Ocimum basillicum L.). Acta Sci. Pol. Hortorum Cultus 9, 3-12.

Bonilla, C. y M. Guerrero. 2010. Albahaca (Ocimum basillicum L.), producción y manejo poscosecha. Corredor Tecnológico Agroindustrial, Cámara de Comercio de Bogotá; Universidad Nacional de Colombia, Bogotá, Colombia.

Box, G. 1954. The exploration and exploitation of the response surfaces: some general considerations and examples. Biometrics 10(1), 16-60. Doi: 10.2307/3001663

Búfalo, J., C. Cantrell., T. Astatkiec, V. Zheljazkov, A. Gawde y C. Fernandes. 2015. Organic versus conventional fertilization effects on sweet basil (Ocimum basillicum L.) growth in a greenhouse system. Ind. Crops Prod. 74, 249-254. Doi: 10.1016/j.indcrop.2015.04.032 
Cenóz, P. y A. Burgos. 2005. Influencia de la fertilización nitrogenada en el rendimiento de la albahaca (Ocimum basillicum L.). Hortic. Argent. 24, 56-57.

Ding, Y., W. Luo y G. Xu. 2006. Characterisation of magnesium nutrition and interaction of magnesium and potassium in rice. Ann. Appl. Biol. 149, 111-123. Doi: 10.1111/j.1744-7348.2006.00080.x

Ferreira, S., L. Bulegon., R. Yassue y M. Echer. 2016. Efeito da adubação nitrogenada e da sazonalidade na produtividade de Ocimum basillicum L. Rev. Bras. Plantas Med. 18(1), 67-73. Doi: 10.1590/1983-084X/15_035

Frabboni, L., G. da Simone y V. Russo. 2011. The influence of different nitrogen treatments on the growth and yield of basil (Ocimum basillicum L.). J. Chem. Chem. Eng. 5, 799-803.

Golcz, A., B. Politycka y K. Seidler-Łożykowska. 2006. The effect of nitrogen fertilization and stage of plant development on the mass and quality of sweet basil leaves (Ocimum basillicum L.). Herba Pol. 52, 22-30.

Hu, W., W. Zhao, J. Yang, D. Oosterhuis, D. Loka y Z. Zhou. 2016. Relationship between potassium fertilization and nitrogen metabolism in the leaf subtending the cotton (Gossypium hirsutum L.) boll during the boll development stage. Plant Physiol. Biochem. 101, 113123. Doi: 10.1016/j.plaphy.2016.01.019

Jaćimović, G., J. Crnobarac., J.T. Ninić., B. Marinković, J. Ninić-Todorović y J. Štetić. 2010. The yield and morphological properties of calendula and basil in relation to nitrogen fertilization. Godina. 34, 69-79.

Jákli, B., E. Tavakola, M. Tränknera, M. Senbayrama y K. Dittert. 2017. Quantitative limitations to photosynthesis in $\mathrm{K}$ deficient sunflower and their implications on water-use efficiency. J Plant Physiol. 209, 20-30. Doi: 10.1016/j.jplph.2016.11.010

Koba, K., P.W. Poutouli., C. Raynaud., J.P. Chaumont y K. Sada. 2009. Chemical composition and antimicrobial properties of different basil essentials oils chemotypes from Togo. Bangladesh J. Pharmacol. 4, 1-8.

Malik, A.A., S. Suryapani y J. Ahmad. 2011. Chemical vs organic cultivation of medicinal and aromatic plants: the choice is clear. Int. J. Med. Arom. Plants 1, 5-13.

Marschner, H. 2002. Mineral nutrition of higher plants. Academic Press, New York, NY, USA.

Matsumoto, S., G. Araujo y A. Viana. 2013. Growth of sweet basil depending on nitrogen and potassium doses. Hortic. Bras. 31(3), 489-493. Doi: 10.1590/ S0102-05362013000300024

Mejía, M. 2010. Conceptos sobre fisiología de absorción y funciones de los minerales en la nutrición de las plantas. Facultad de Ciencias Agropecuarias, Universidad Nacional de Colombia, Palmira, Colombia.
Nguyen, P.M.N., E.M. Kwee y E.D. Niemeyer. 2010. Potassium rate alters the antioxidant capacity and phenolic concentration of basil (Ocimum basillicum L.). Food Chem. 123, 1235-1241. Doi: 10.1016/j. foodchem.2010.05.092

Nurzyńska-Wierdak, R., E. Rożek., K. Dzida y B. Borowski. 2012. Growth response to nitrogen and potassium fertilization of common basil (Ocimum basillicum L.) plants. Acta Sci. Pol. Hortorum Cultus 11(2), 275-288.

Pacheco, A. y A. Jürgen. 2005. Plantas aromáticas como cultivo intercalado, experiencias y efectos alelopáticos sobre el café (Coffea arabica L.). pp. 207-216. En: Memorias I Congreso Internacional de Plantas Medicinales en Villahermosa. Tabasco, México.

Palencia, S.G., T. Mercado-Fernández, y E. Combatt-Caballero. 2006. Estudio agroclimático del departamento de Córdoba, Universidad de Cordoba, Montería, Colombia.

Patil, N.M. 2010. Biofertilizer effect on growth, protein and carbohydrate content in Stevia rebaudiana var. Bertoni. Rec. Res. Sci. Tecnol. 2(10), 42-44.

Pereyra, M. 2002. Asimilación del nitrógeno en plantas. En: http://exa.unne.edu.ar/biologia/fisiologia.vegetal/ Asimilacion\%20del\%20nitrogeno.pdf; consulta: marzo de 2017.

Pessarakli, M. (ed.) 2001. Handbook of plant and crop physiology. $2^{\text {nd }}$ ed. CRC Press, Boca Raton, FL, USA. Doi: $10.1201 / 9780203908426$

R Development Core Team. 2015. R: A language and environment for statistical computing Version. 3.2.2. $\mathrm{R}$ Foundation for Statistical Computing, Vienna, Austria.

Rincón, L., A. Pérez, C. Pellicer, J. Sáenz y A. Abadía. 2002. Influencia de la fertilización nitrogenada en la absorción de nitrógeno y acumulación de nitratos en la lechuga iceberg. Inv. Agr.: Prod. Prot. Veg. 17(2), 303-318.

Robertson, G.P. y P.M. Vitousek. 2009. Nitrogen in agriculture: balancing the cost of an essential resource. Ann. Rev. Environ. Resour. 34, 97-125. Doi: 10.1146/annurev.environ.032108.105046

Sharafzadeh, S. y O. Alizadeh. 2011. Nutrient supply and fertilization of basil. Adv. Environ. Biol. 5, 956- 960.

Sifola, M.I. y G. Barbieri. 2006. Growth, yield and essential oil content of three cultivars of basil grown under different levels of nitrogen in the field. Sci. Hort. 108, 408-413. Doi: 10.1016/j.scienta.2006.02.002

Taiz, L. y E. Zeiger, 2006. Plant physiology. $4^{\text {th }}$ ed. Sinauer Associates, Sunderland, MA, USA.

Wahab, A.S.A. y L. Hornok. 1982. Effect of NPK fertilization on Ocimum basillicum yield and essential oil content. Kert. Egyet. Közlem. 45, 65-73. 
Wang, N., H. Hua., A. Egrinya Eneji., Z. Li., L. Duan y X. Tian. 2012. Genotypic variations in photosynthetic and physiological adjustment to potassium deficiency in cotton (Gossypium hirsutum). J. Photochem. Photobiol. B. 110, 1-8. Doi: 10.1016/j.jphotobiol.2012.02.002
Zheljazkov, V., C. Cantrell, B. Tekwani y S. Khan. 2008. Content, composition, and bioactivity of the essential oils of three basil genotypes as a function of harvesting. J. Agric. Food Chem. 56(2), 380-385. Doi: 10.1021/jf0725629 